\title{
Avaliação da efetividade do controle da hipertensão arterial em unidade básica de saúde
}

\section{Evaluation of the effectiveness of arterial hypertension control in a primary health unit}

\author{
Arnaldo Sala, Alexandre Nemes Filho e José Eluf-Neto \\ Departamento de Medicina Preventiva da Faculdade de Medicina da Universidade de São Paulo. \\ São Paulo, SP - Brasil
}

\begin{abstract}
Resumo
Como parte integrante da avaliação de desempenho do Programa de Atenção à Saúde no Envelhecimento, desenvolvido em uma unidade básica de saúde, foi mensurada a efetividade da hipertensão arterial, segundo a redução dos níveis de pressão arterial em indivíduos hipertensos submetidos a ações programáticas para controle da doença, procurando identificar condições associadas com tal redução. Dos 396 pacientes portadores de hipertensão arterial sistêmica inscritos no Programa, no período de 01/01/92 a 30/06/93, foram considerados para esta avaliação 250 casos que apresentavam, além de níveis pressóricos elevados (PA $\geq 160 / 95 \mathrm{mmHg}$ ) em atendimentos iniciais no serviço (anteriores à inscrição no programa), pelo menos duas consultas médicas no seguimento programático. As diferenças de níveis pressóricos entre as medidas realizadas nas consultas anteriores ao início do atendimento programático, e as realizadas a partir do início destes atendimentos foram analisadas segundo o nível pressórico inicial, idade, sexo, diagnósticos na inscrição e faltas ao agendamento programático. Obteve-se redução na pressão arterial diastólica (PAD) de $5 \mathrm{mmHg}$ ou mais, e/ou redução de $10 \mathrm{mmHg}$ ou mais na pressão arterial sistólica (PAS) em 197 (78,8\%) pacientes. A média da redução da PAD foi $8,8 \mathrm{mmHg}$ (d.p. = 11,4), e da PAS foi 17,7 mmHg (d.p. = 18,6). Resultados de diversos estudos epidemiológicos permitem inferir redução do risco de mortalidade por doença cardiovascular em proporção considerável de indivíduos inscritos no Programa. Em 111 (44,4\%) indivíduos ocorreu normalização da pressão aos níveis preconizados pelo Programa. A análise por meio de regressão linear múltipla demonstrou que, entre as variáveis estudadas, a pressão inicial e a percentagem de faltas no seguimento programático estiveram associadas de modo independente com a redução da PAS e da PAD. A idade esteve associada independentemente apenas com a redução da PAS. A participação da idade e da percentagem de faltas no seguimento programático revelam que o resultado final do trabalho programático não é insensível aos diferentes modos com que as pessoas assumem o cuidado com a própria saúde.
\end{abstract}

Avaliação de programas. Hipertensão, prevenção \& controle.

Correspondência para / Correspondence to: Arnaldo Sala - Centro de Saúde Escola Prof. Samuel B. Pessoa. Av. Vital Brasil, 1490 - $05503-000$ São Paulo, SP - Brasil. Fax: (011) 212-1690 E-mail: arnasala@usp.br

Edição subvencionada pela FAPESP. Processo 95/2290-6.

Recebido em 4.5.1995. Aprovado em 23.10.1995. 


\begin{abstract}
The present study is part of a performance evaluation of the Aging Health Care Program carried out at a Primary Health Care Clinic. Activities for Arterial Hypertension (AH), control oriented accorging to a risk assessment, in conformity with Ministry of Health norms, are included in the Program mentioned. The effectiveness of the reduction of blood pressure (BP) levels in hypertensive individuals subjected to programmatic activities for disease control, in an attempt to identify conditions associated with that reduction, is evaluated. Of the 396 patients with AH registered under the Program between 01/01/92 and 06/30/93, 250 cases were included for this evaluation. These patients had, in addition to high blood pressure levels (BP $\geq 160 / 95 \mathrm{~mm} \mathrm{Hg}$ ) on the first attendances at the health center (before registration in the programme), at least two medical consultations during program follow-up. The differences in blood pressure levels between measurements taken during consultations before the beginning of attendance at the program and those taken on the first attendances to the programme were analysed according to initial blood pressure level, age, sex, registering diagnosis and absences during the program follow-up. Reductions of $\geq 5 \mathrm{~mm} \mathrm{Hg}$ in diastolic blood pressure (DBP) and/or $\geq 10 \mathrm{~mm} \mathrm{Hg}$ in systolic blood pressure (SBP) were obtained in 197 (78.8\%) patients. The mean reduction was $8.8 \mathrm{~mm} \mathrm{Hg}$ ( $\mathrm{sd}=$ 11.4) for DBP and $17.7 \mathrm{~mm} \mathrm{Hg}$ ( $\mathrm{md}=18.6$ ) for SBP. Results of several epidemiological studies allow the inference that a decrease in the mortality risk from Cardiovascular Disease can be achieved in a significant proportion of the individuals registered in the program. Blood pressure normalization according to program norms occurred in 111 (44.4\%) individuals. Multiple linear regression analysis demonstrated that initial blood pressure and percentages of absenteeism during the follow-up were independently associated with the reductions in SBP and DBP. Age was independently associated only with the reductions in SBP. Participation of age and percentage of absenteeism during follow-up thus reveal that the final result of the programmed work is also a result of the different ways people take care of their own health.
\end{abstract}

Program evaluation. Hypertension, prevention and control.

\section{INTRODUÇÃO}

No trabalho realizado em unidade básica de saúde, as ações em saúde denominadas no presente texto como Ações Programáticas (ou Programas em Saúde) referem-se a um conjunto articulado de atividades voltadas para uma população-alvo tendo dupla dimensão de fazer incidir, simultaneamente, seu objeto de preocupações sobre o indivíduo e sobre o coletivo ${ }^{14}$.

No serviço analisado, as ações dirigidas ao controle da Hipertensão Arterial Sistêmica (HAS) têm assumido as características de Ações Programáticas na medida em que são desenvolvidos cuidados, dirigidos ao indivíduo (através do controle de níveis pressóricos e de alguns outros fatores de risco para doença cardiovascular - DCV), por meio do atendimento clínico, dentro de uma racionalidade epide- miológica cujo objetivo final é a redução da morbimortalidade por DCV em uma população-alvo (através do controle dos fatores de risco individuais e de medidas que incidem imediatamente sobre o coletivo, tais como ações educativas).

A avaliação de um programa de controle de DCV, baseado em ações de controle da HAS por meio do atendimento clínico individual, deveria apreciar seus efeitos em termos da redução da morbimortalidade por este grupo de doenças na população. Porém, dificuldades conceituais e operacionais tornam nem sempre possíveis, na prática, a realização de tal procedimento. Uma primeira dificuldade é que os resultados sofrem os efeitos de todos os outros fatores não abrangidos pelo programa; deste modo, uma modificação no perfil epidemiológico das DCVs não poderia ser exclusivamente atribuída ao Programa. Em segundo lugar, o grupo de indivíduos com alto risco 
para DCV não produz a maioria dos casos de doença, na medida em que é um grupo numericamente bem menor que o dos indivíduos de risco mais baixo, como os hipertensos leves. (Vale lembrar também os casos ocorridos entre os indivíduos não hipertensos, que, portanto, não são considerados pelo Programa). O efeito obtido pelo Programa estaria sempre "diluído" nos índices de DCV da população não hipertensa, mas provavelmente portadora de outros fatores de risco. Por fim, a obtenção do resultado desejado na população não se faz de imediato, mas após um período de vários anos, o que dificulta processos de reorientação e aperfeiçoamento das ações contidas no Programa.

Assim, uma metodologia de avaliação, que possibilite apreender elementos do processo de trabalho que envolve o atendimento dos indivíduos de risco (no caso, portadores de HAS), parece ser pertinente, em especial na situação em que já é bem estabelecida uma conexão entre redução de níveis pressóricos e redução das DCVs.

A HAS pode ser considerada como uma doença peculiar, na medida em que não foi devido a reiterado sofrimento individual, que a doença trouxesse aos seus portadores, o fator determinante dela terse tornado objeto de preocupação da medicina clínica. Ao contrário, por ser, de fato, uma doença freqüentemente assintomática, a HAS foi definitivamente incluída na nosologia médica a partir de diversos estudos epidemiológicos que mostraram maior morbidade e mortalidade por DCV entre os indivíduos com pressão arterial sistólica e/ou diastólica elevada ${ }^{3,8}$. Do mesmo modo, ensaios clínicos têm confirmado esses resultados, ao indicarem redução da morbimortalidade por DCV em indivíduos que obtiveram redução terapêutica nos níveis pressóricos ${ }^{1}$.

A DCV tem sido a principal causa de óbito, tanto nos países chamados em desenvolvimento quanto nos mais desenvolvidos. Nestes últimos, tem se observado diminuição de suas taxas há vários anos, sendo esta atribuída tanto ao melhor controle da HAS quanto à redução de outros fatores de risco cardiovascular $^{2,15,16,17}$. Mesmo na cidade de São Paulo observa-se, em anos recentes, redução da mortalidade por doença coronariana ${ }^{6}$ e por doença cerebrovascular ${ }^{7}$.

A literatura tem apontado uma relação contínua entre os níveis pressóricos de uma dada população e as taxas de mortalidade por DCV, assinalando que uma redução média de $5 \mathrm{mmHg}$ na pressão arterial diastólica (e/ou de $10 \mathrm{mmHg}$ na pressão arterial sistólica) diminui o risco de acidente vascular cere- bral em aproximadamente um terço, e o risco de coronariopatia em cerca de um sexto ${ }^{3}$.

As proposições de redução da morbimortalidade por DCV passam, então, pela diminuição dos níveis pressóricos (sistólico e diastólico) através de estratégias, sejam elas por meio de ações dirigidas a toda população, sejam através de ações dirigidas aos grupos considerados de risco, tais como os portadores de hipertensão arterial $^{12}$.

Na medida em que a maioria dos óbitos por DCV, atribuídos a HAS, ocorre em indivíduos com hipertensão arterial leve (por ser este grupo numericamente bem maior que o dos hipertensos graves), parece adequado a uma rede básica de saúde a tarefa de controle da grande maioria desses indivíduos e o procedimento de avaliação da sua efetividade.

O objetivo do presente estudo é analisar a efetividade do controle dos níveis pressóricos em pacientes portadores de HAS, submetidos a um seguimento ambulatorial constituído a partir de Ações Programáticas específicas, desenvolvidas em uma unidade básica de saúde.

\section{METODOLOGIA}

A unidade básica de saúde estudada (UBS-SP) está localizada no Município de São Paulo, no bairro do Butantã, e atende a uma população geograficamente delimitada, constituída por 47.016 habitantes, em 1991 (censo 1991, IBGE, dados por setor censitário), da qual estima-se que 60,4\% têm 20 anos de idade ou mais.

Na UBS-SP, as ações de controle de DCV, e mais especificamente aquelas relacionadas ao controle da HAS, seguem a padronização do Ministério da Saúde que estabelece como critério diagnóstico de hipertensão arterial a medida de pressão arterial sistólica igual ou superior a $160 \mathrm{mmHg}$ e/ou pressão arterial diastólica igual ou superior a $95 \mathrm{mmHg}$, em pelo menos duas verificações realizadas em dias diferentes, em indivíduos de 20 anos ou mais $^{9,10}$.

A medida de pressão arterial na unidade é usualmente feita durante a consulta médica, com esfigmomanômetro aneróide, obtida com o paciente sentado, após 2 a 5 minutos de repouso, e registrada de preferência no braço direito para comparação subseqüente. A pressão sistólica corresponde aos primeiros ruídos arteriais (fase I dos sons de Korotkoff) com a desinsuflação do manguito, e a pressão diastólica corresponde ao seu desaparecimento (fase $\mathrm{V}$ dos sons de Korotkoff).

A organização dos cuidados dirigidos aos pacientes portadores de doença crônico-degenerativa possui, na UBS-SP, dois momentos principais: o atendimento não programático e o acompanhamento médico programático. O primeiro, ou Pronto-Atendimento, tem dois objetivos básicos: o atendimento médico imediato aos pacientes que procuram o serviço sem consulta agendada e com queixa, 
e a captação ativa para as atividades programáticas. $\mathrm{O}$ acompanhamento médico programático envolve consultas médicas agendadas e atendimentos em grupo de cunho educativo e vivencial.

Para as ações de controle da HAS, a captação ativa da demanda é realizada no Pronto-Atendimento através da medida rotineira da pressão arterial de todos os indivíduos, independentemente da queixa que apresentam. Também é verificada a pressão arterial de todos os pacientes que fazem seguimento no serviço, em qualquer um dos programas, pelo menos uma vez por ano. O paciente considerado hipertenso é então encaminhado para consulta de início de seguimento (que corresponde à inscrição no programa). Nessa consulta são abordados, além da hipertensão, outros fatores de risco aos quais o indivíduo estaria submetido. A seguir, o paciente entra numa rotina de seguimento médico trimestral, com realização anual de exames complementares, com o objetivo principal de detectar complicações, bem como diagnosticar, de forma precoce, alguns outros fatores de risco para DCV.

O presente estudo foi constituído por pacientes hipertensos atendidos na UBS-SP, inscritos no Programa de Atenção à Saúde no Envelhecimento, no período compreendido entre $1^{\circ}$ de janeiro de 1992 e 30 de junho de 1993. Trezentos e noventa e seis (396) indivíduos receberam diagnóstico de HAS. Para a presente análise foram selecionados os 250 indivíduos que apresentaram níveis pressóricos elevados nos atendimentos que precederam à consulta que corresponde à inscrição no Programa (uma vez que parte dos pacientes iniciou tratamento e controle dos níveis pressóricos em outro serviço), e com pelo menos duas consultas médicas no seguimento programático (foram considerados todos os atendimentos realizados por estes pacientes desde o início do período citado, até 31 de dezembro de 1993). Esses 250 casos geraram um total de 2.092 atendimentos (além de 406 faltas ao agendamento), de onde foram coletados os dados necessários para a análise da evolução dos níveis pressóricos.

A análise dos dados buscou verificar a ocorrência de controle dos níveis pressóricos de cada paciente inscrito no Programa. As médias das pressões arteriais medidas nos dois primeiros atendimentos anteriores à consulta de inscrição (atendimentos iniciais) foram comparadas com as médias das pressões medidas nos atendimentos realizados a partir da consulta inicial do Programa. Em 139 (55,6\%) dos pacientes houve um único atendimento inicial. Nesses casos foi considerada esta como única medida.

Os resultados, em termos de evolução dos níveis pressóricos, consideraram as diferenças entre as médias pressóricas obtidas nos atendimentos iniciais e as verificadas no seguimento programático, para cada paciente em estudo. Avaliou-se, entre os casos estudados, a redução de pelo menos $5 \mathrm{mmHg}$ na pressão arterial diastólica (PAD) e de pelo menos $10 \mathrm{mmHg}$ na pressão sistólica (PAS), bem como a normalização da pressão arterial aos níveis considerados satisfatórios pelo programa (PA menor que 160 x 95 mmHg). Esta redução e/ou normalização da PA está associada à redução do risco cardiovascular ${ }^{1,3}$.

Utilizou-se o teste de $\mathrm{x}^{2}$ para a comparação de proporções. Na análise da evolução das pressões arteriais sistólica e diastólica, segundo as respectivas pressões iniciais, empregou-se o teste de Kruskal-Wallis.

Considerando que a maior parte das variáveis em estudo é de tipo contínuo, optou-se por uma análise de regressão linear. Primeiramente, procedeu-se à regressão linear simples, tomando como variável dependente a redução da PAD, com cada uma das variáveis independentes estudadas (PAD inicial, sexo, idade, diagnóstico na inscrição no Programa e percentagem de faltas no seguimento programático). Posteriormente utilizou-se regressão linear múltipla por acréscimo das variáveis uma a uma. As variáveis categóricas (sexo e diagnóstico) foram transformadas em variáveis dummy. Procedimento análogo foi realizado para análise da $\mathrm{PAS}^{5}$.

\section{RESULTADOS}

A maioria dos pacientes estudados foi do sexo feminino - 172 casos $(68,8 \%)$. A média de idade dos indivíduos do sexo masculino (58,2 anos), embora superior a dos sexo feminino (55,3 anos), não apresentou diferença significante $(\mathrm{p}=0,08)$. Mais da metade dos homens incluídos apresentou idade igual ou superior a 60 anos (Tabela 1).

Os resultados apresentados nas Tabelas 2 e 3, relativos a pressão arterial encontrada nos atendimentos anteriores às consultas programáticas, indicaram maior número de casos com hipertensão diastólica isolada (65 casos) do que com hipertensão sistólica isolada (27 casos). Além disso, mostraram uma associação direta entre idade e níveis pressóricos diastólico e sistólico. A proporção de indivíduos com PAD normal (hipertensão sistólica isolada) foi claramente maior nos indivíduos com 60 anos ou mais

Tabela 1 - Distribuição dos pacientes segundo sexo e idade.

\begin{tabular}{|c|c|c|c|c|c|c|}
\hline \multirow{3}{*}{$\begin{array}{l}\text { Faixa etária } \\
\text { (em anos) }\end{array}$} & \multicolumn{4}{|c|}{ Sexo } & \multirow{2}{*}{\multicolumn{2}{|c|}{ Total }} \\
\hline & \multicolumn{2}{|c|}{ Masculino } & \multicolumn{2}{|c|}{ Feminino } & & \\
\hline & $\mathrm{n}^{\mathrm{o}}$ & $\%$ & $\mathrm{n}^{\mathrm{o}}$ & $\%$ & $\mathrm{n}^{\mathrm{o}}$ & $\%$ \\
\hline$<50$ & 16 & 20,5 & 53 & 30,8 & 69 & 27,6 \\
\hline 50 a 59 & 20 & 25,6 & 62 & 36,0 & 82 & 32,8 \\
\hline$\geq 60$ & 42 & 53,8 & 57 & 33,1 & 99 & 39,6 \\
\hline Total & 78 & 100,0 & 172 & 100,0 & 250 & 100,0 \\
\hline
\end{tabular}

$x^{2}=9,7$ (2 G.L.), $p=0,0080$. 
Tabela 2 - Distribuição dos pacientes segundo idade e pressão arterial diastólica inicial.

\begin{tabular}{lcccc}
\hline Faixa etária & \multicolumn{3}{c}{ PAD inicial $(\mathrm{em} \mathrm{mmH})$} & \\
\cline { 2 - 4 } (em anos) & $<95$ & $95-114$ & $\geq 115$ & Total \\
\hline$<50$. & 1 & 59 & 9 & 69 \\
50 a 59 & 5 & 61 & 16 & 82 \\
$\geq 60$ & 21 & 66 & 12 & 99 \\
\hline Total & 27 & 186 & 37 & 250 \\
\hline $\mathrm{x}^{2}=21,0$ (4 G.L.), $\mathrm{p}=0,0003$. & & & &
\end{tabular}

(Tabela 2), enquanto a proporção de indivíduos com PAS normal (hipertensão diastólica isolada) diminui com o aumento da idade (Tabela 3).

Cento e sessenta dos indivíduos $(64,0 \%)$ apresentaram diagnóstico de HAS exclusiva, 32 (12,8\%) de HAS e diabetes mellitus e $58(23,2 \%)$ apresentaram outros diagnósticos associados a HAS (cardiopatia, doença pulmonar obstrutiva crônica e obesidade).

A média de redução da $\operatorname{PAD}(8,8 \mathrm{mmHg})$ foi significantemente maior nos indivíduos com PAD inicial elevada ( $\mathrm{p}<0,0001$ ) (Tabela 4). Os pacientes com hipertensão sistólica isolada praticamente não apresentaram alteração (aumento médio de $0,4 \mathrm{mmHg}$ ). A redução da PAS também esteve associada aos níveis pressóricos iniciais (Tabela 5), mas, diferentemente do ocorrido com a PAD, mesmo pacientes com PAS inicial "normal" $(<160$ $\mathrm{mmHg}$ ) apresentaram redução (média de redução $=5,8$ $\mathrm{mmHg})$.

Dos 250 casos em estudo, 197 (78,8\%) apresentaram redução da PAD de $5 \mathrm{mmHg}$ ou mais, e/ou redução da PAS de $10 \mathrm{mmHg}$ ou mais. Em 111 casos (44,4\% do to-
Tabela 3 - Distribuição dos pacientes segundo idade e pressão arterial sistólica inicial.

\begin{tabular}{lcccc}
\hline Faixa etária & \multicolumn{3}{c}{ PAS inicial $(\mathrm{em} \mathrm{mmHg})$} & \\
\cline { 2 - 4 } (em anos) & $<160$ & $160-179$ & $\geq 180$ & Total \\
\hline$<50$. & 30 & 28 & 11 & 69 \\
50 a 59 & 20 & 36 & 26 & 82 \\
$\geq 60$ & 15 & 47 & 37 & 99 \\
\hline Total & 65 & 111 & 74 & 250 \\
\hline $\mathrm{x}^{2}=19,6$ (4 G.L.), $\mathrm{p}=0,0006$. & & & &
\end{tabular}

tal) obteve-se normalização da pressão arterial a níveis considerados normais pelo Programa (PAS $<160$ e PAD $<95)$. Dos pacientes restantes, 25 (10,0\% do total) mantiveram níveis pressóricos altos ( $\geq 180 \times 115 \mathrm{mmHg})$.

Foram analisadas as médias pressóricas das consultas iniciais, da consulta de inscrição e das consultas programáticas subseqüentes; verificou-se que a redução da pressão ocorreu sobretudo entre os atendimentos iniciais e a inscrição no programa: em 192 casos (76,8\%) obteve-se, já na consulta de inscrição, redução na PAD de $5 \mathrm{mmHg}$ ou mais, e/ou redução na PAS de $10 \mathrm{mmHg}$ ou mais, com normalização da pressão arterial em 95 pacientes $(38,0 \%)$. Não se observou, contudo, piora nos níveis pressóricos entre os pacientes em seguimento programático mais prolongado (mais de um ano).

A evolução da PAD e da PAS foi analisada, por regressão linear, segundo as variáveis pressão inicial, sexo, idade, diagnóstico na inscrição ao Programa e percentagem de faltas no seguimento programático.

Na regressão linear simples, a redução da PAD esteve significantemente associada apenas com a pressão diastó-

Tabela 4 - Evolução da pressão arterial diastólica (PAD) segundo pressão inicial.

\begin{tabular}{lcccccc}
\hline \multirow{2}{*}{$\begin{array}{l}\text { PAD inicial } \\
\text { (em mmHg) }\end{array}$} & \multicolumn{3}{c}{ Evolução da PAD } & & DP \\
\cline { 2 - 5 } & $\begin{array}{c}\text { Piora ou } \\
\text { Redução } \\
<5 \mathrm{~mm} \mathrm{Hg}\end{array}$ & $\begin{array}{c}\text { Redução } \\
\geq 5 \mathrm{mmHg}\end{array}$ & $\begin{array}{c}\text { Média de } \\
\text { Redução } \\
\text { (em mm Hg) }\end{array}$ & & $\mathrm{N}$ \\
\hline$<95^{*}$ & 18 & 9 & $-0,4$ & 9,6 & 27 \\
95 a 114 & 70 & 116 & 8,1 & 9,7 & 186 \\
$\geq 115$ & 6 & 31 & 19,2 & 13,5 & 37 \\
\hline Total & 94 & 156 & 8,8 & 11,4 & 250 \\
\hline
\end{tabular}

* hipertensão arterial sistólica isolada.

Kruskal-Wallis = 40,3 (2 G.L.), p < 0,0001.

$\mathrm{DP}=$ Desvio-Padrão

Tabela 5 - Evolução da pressão arterial sistólica (PAS) segundo pressão inicial.

\begin{tabular}{lcccccc}
\hline \multirow{2}{*}{$\begin{array}{l}\text { PAS inicial } \\
\text { (em mmHg) }\end{array}$} & \multicolumn{3}{c}{ Evolução da PAS } & & DP \\
\cline { 2 - 5 } & $\begin{array}{c}\text { Piora ou } \\
\text { Redução } \\
<10 \mathrm{~mm} \mathrm{Hg}\end{array}$ & $\begin{array}{c}\text { Redução } \\
\geq 10 \text { mmHg }\end{array}$ & $\begin{array}{c}\text { Média de } \\
\text { Redução } \\
\text { (em mmHg) }\end{array}$ & & $\mathrm{N}$ \\
\hline$<160^{*}$ & 37 & 28 & 5,8 & 13,0 & 65 \\
160 a 179 & 31 & 80 & 16,7 & 13,6 & 111 \\
$\geq 180$ & 10 & 64 & 29,6 & 22,0 & 74 \\
\hline Total & 78 & 172 & 17,7 & 18,6 & 250 \\
\hline
\end{tabular}

* hipertensão arterial diastólica isolada.

Kruskal-Wallis $=60,6$ (2 G.L.), $\mathrm{p}<0,0001$.

DP $=$ Desvio-Padrão 
lica inicial $\left(\mathrm{r}^{2}=0,32,0,48<\mathrm{R}<0,65, \mathrm{~b}=0,57\right)$. Na regressão linear múltipla, a inclusão das variáveis idade e percentagem de faltas melhorou o poder de explicação da variável dependente, obtendo-se, assim, $\mathrm{r}^{2}=0,38 \mathrm{com}$ a seguinte equação de regressão:

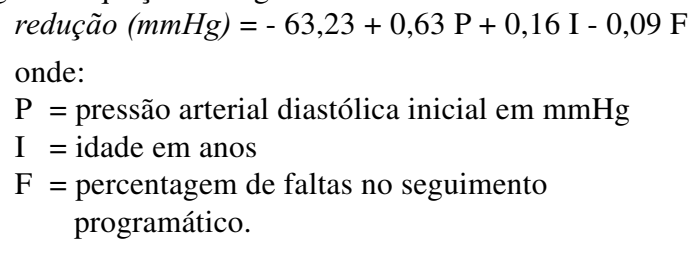

Isto significa, por exemplo, que, controladas as outras variáveis analisadas, os indivíduos que não faltaram no seguimento apresentariam uma redução na PAD de 4,5 $\mathrm{mmHg}$ a mais que indivíduos que faltaram a $50 \%$ dos atendimentos; de modo análogo, a redução na PAD seria de 3,2 $\mathrm{mmHg}$ a mais nos indivíduos de 70 anos, em relação àqueles com 50 anos de idade.

Na análise da evolução da pressão arterial sistólica, a regressão linear simples mostrou associação estatisticamente significante apenas com a pressão arterial sistólica inicial $\left(r^{2}=0,38,0,53<R<0,69, b=0,53\right)$. Na regressão múltipla, somente a inclusão da variável percentagem de faltas melhorou o poder de explicação da redução da pressão arterial sistólica, obtendo-se, $\mathrm{r}^{2}=0,40$, com a seguinte equação de regressão:

redução $(\mathrm{mmHg})=-70,35+0,53 \mathrm{P}-0,12 \mathrm{~F}$

onde:

$\mathrm{P}=$ pressão arterial sistólica inicial em $\mathrm{mmHg}$

$\mathrm{F}=$ percentagem de faltas no seguimento programático.

$\mathrm{Na}$ regressão linear simples verificou-se associação negativa entre idade e percentagem de faltas $(r=-0,18)$. A idade apresentou, ainda, associação negativa com a PAD inicial $(r=-0,17)$, mas positiva com a PAS inicial $(r=$ $0,19)$. O sexo e o diagnóstico inicial não apresentaram associação significante com a redução da PAS ou da PAD.

\section{DISCUSSÃO}

O presente estudo possibilitou avaliar a efetividade de ações em saúde dirigidas ao controle da HAS, organizadas segundo a perspectiva do "enfoque de risco" para o controle da doença cardiovascular na população, a partir de serviços oferecidos em uma unidade básica de saúde.

Quase $80 \%$ dos pacientes estudados apresentaram diminuição da PAD de $5 \mathrm{mmHg}$ ou mais e/ou diminuição da PAS de $10 \mathrm{mmHg}$ ou mais. Apesar de algumas dúvidas relativas ao possível benefício do tratamento da hipertensão arterial $^{2}$, resultados mais recentes, quando corrigidos quanto à classificação incorreta dos níveis pressóricos, mostram redução importante da morbimortalidade por DCV ${ }^{1,8}$. Em quase metade dos pacientes $(44,4 \%)$ obteve-se normalização das pressões sistólica e diastólica.
As maiores quedas de PA foram observadas nos pacientes com pressão inicial mais elevada (tanto sistólica quanto diastólica). Resultados semelhantes têm sido encontrados em diversos estudos ${ }^{4,11}$. No tocante à PAS, mesmo nos indivíduos com pressão inferior a $160 \mathrm{mmHg}$ observou-se redução dos níveis pressóricos. Estes resultados permitem inferir a existência de um benefício real do seguimento programático, seja no grupo com PA mais elevada (portanto de maior risco de complicações e óbito), seja no grupo com hipertensão arterial leve (que reúne o maior número de casos).

Os resultados mostraram que é sobretudo nos atendimentos iniciais que efetivamente ocorreu redução na pressão arterial; portanto, coube às consultas programáticas a função principal de manutenção dos níveis pressóricos já reduzidos.

Em termos de efetividade no controle da HAS, deve-se apontar que em 55,6\% dos casos não se obteve normalização dos níveis pressóricos (embora a maior parte apresentasse redução da PAS e/ou da PAD) e $10,0 \%$ mantiveram níveis pressóricos altos $(\mathrm{PA} \geq 180 \times 115)$.

$\mathrm{Na}$ análise multivariada referente à pressão diastólica, além da PA inicial, foi possível evidenciar também a contribuição da idade e da percentagem de faltas no seguimento, na redução da PAD. Indivíduos com idade mais elevada e com menor proporção de faltas apresentaram redução mais intensa da PAD. Na redução da PAS, além da PA inicial, hove contribuição significativa da percentagem de faltas no seguimento.

Trabalho anterior realizado na mesma unidade apresentava perda de $19,7 \%$ dos pacientes hipertensos não portadores de outras patologias crônicodegenerativas entre o pronto-atendimento e a inscrição no Programa ${ }^{13}$. Esse mesmo trabalho mostrava também maior dificuldade na inscrição de indivíduos do sexo masculino e de hipertensos sem outra patologia crônico-degenerativa. Por outro lado, o presente trabalho não encontrou relação significante entre sexo e diagnóstico com a redução da pressão arterial; mas demonstrou que, aqueles que efetivamente aderiram a uma proposta de seguimento, em uma unidade básica de saúde, obtiveram resposta terapêutica favorável.

A verificação de que a proporção de faltas, um indicador de utilização do serviço pela clientela, participa no resultado final do controle dos níveis de pressão arterial sugere duas hipóteses, não excludentes entre si: 1) a presença do paciente na unidade de saúde é determinante na evolução favorável do controle da HAS; 2) fatores determinantes do controle favorável da HAS (por exemplo, aque- 
les relativos às possibilidades ou às motivações individuais que levam a atitudes que contribuem para a redução da pressão arterial) estão associados também a uma maior utilização do serviço de saúde. Em outros termos, a participação da idade e da percentagem de faltas no seguimento, como fatores independentes na equação de regressão, revela que o resultado final do trabalho programático não é insensível aos diferentes modos com que as pessoas assumem o cuidado com a própria saúde. Embora o presente estudo não tenha pretendido apreender os determinantes socioculturais dos comportamentos e atitudes de uma clientela frente a uma proposta de cuidados em saúde, baseados no conceito de ris-

\section{REFERÊNCIAS BIBLIOGRÁFICAS}

1. COLLINS, R. et al. Blood pressure, stroke and coronary heart disease. Part 2, short-term reductions in blood pressure: overview of randomised drug trials in their epidemiological context. Lancet, 335:827-38, 1990.

2. ELUF NETO, J. et al. Tratamento da hipertensão e declínio da mortalidade por acidentes vasculares cerebrais. Rev. Saúde Pública, 24:332-6, 1990.

3. GUIDELINES Sub-Committee of the WHO/ISH Mild Hypertension Liaison Committee: 1993 guidelines for the management of mild hypertension: memorandum from a WHO/ISH meeting. Bull World Health Organ., 71: 503-17, 1993.

4. HYPERTENSION Detection and Follow-up Program Cooperative Group. Five-year findings of the hypertension detection and follow-up program. 1- Reduction in mortality of persons with high blood pressure, including mild hypertension. JAMA, 242:2562-71, 1979.

5. KIRKWOOD, B.R. Essentials of medical statistics. London, Blackwell Scientific Publications, 1988.

6. LOLIO, C.A. \& LAURENTI, R. Mortalidade por doença isquêmica do coração no município de São Paulo: evolução de 1950 a 1981 e mudanças recentes na tendência. Arq. Bras. Cardiol., 46:153-6, 1986.

7. LOLIO, C. A.\& LAURENTI, R. Tendência da mortalidade por doenças cerebrovasculares em adultos maiores de 20 anos no município de São Paulo (Brasil), 1950 a 1981. Rev. Saúde Pública, 20:343-6, 1986.

8. McMAHON, S. et al. Blood pressure, stroke, and coronary heart disease - Part 1, prolonged differences in blood pressure: prospective observational studies corrected for the regression dilution bias. Lancet, 335:765-74, 1990. co, parece razoável supor que modificações no serviço para obtenção de melhores resultados programáticos não se esgotem em medidas que visem apenas à redução das faltas ao serviço. A correlação presente entre idade e percentagem de faltas atesta a necessidade de compreensão mais ampliada desta questão.

\section{AGRADECIMENTOS}

Ao Prof. Naim Sauaia, do Departamento de Medicina Preventiva da Faculdade de Medicina da USP, pelo apoio na análise estatística.

9. MINISTÉRIO DA SAÚDE. Normas técnicas para o programa nacional de educação e controle da hipertensão arterial (PNECHA). Brasília, Centro de Documentação, 1988.

10. MINISTÉRIO DA SAÚDE. Controle da hipertensão - uma proposta de integração ensino serviço. Brasília, $\mathrm{CDCV} /$ NUTES, 1993.

11. REPORT by the Management Committee. The Australian therapeutic trial in mild hypertension. Lancet, 1:1261-7, 1980.

12. ROSE, G. Indivíduos enfermos y poblaciones enfermas. In: Buck, C. et al. El desafio de la epidemiologia: problemas y lecturas seleccionadas. Washington, D. C. Organización Panamericana de Salud, 1988. p. 900-9 (OPS - Publicación científica, 505).

13. SALA, A. et al. Avaliação do processo de atendimento a pacientes portadores de doença crônico-degenerativa em uma unidade básica de saúde. Rev. Saúde Pública, 27:46371, 1993.

14. SCHRAIBER, L.B. Programação em saúde hoje. São Paulo, HUCITEC, 1990.

15. WALKER, W.J. Changing United States life-style and declining vascular mortality: cause or coincidence? N. Engl. J. Med., 297:163-5, 1977.

16. WALKER, W. J. Changing United States life-style and declining vascular mortality - a retrospective. N. Engl. J. Med., 308:659-61, 1988.

17. UEMURA, K. \& PISA, Z. Recent trends in cardiovascular diseases mortality in 27 industrialized countries. World Health Statist. Quart., 38:148-62, 1985. 